\title{
Uso de Álcool, Drogas, Níveis de Impulsividade e Agressividade em Adolescentes do Rio Grande do Sul
}

\author{
Rosa Maria Martins de Almeida \\ Laís Broch Trentini \\ Universidade Federal do Rio Grande do Sul \\ Porto Alegre, RS, Brasil \\ Lidiane Andreza Klein \\ Universidade do Vale do Rio dos Sinos \\ São Leopoldo, RS, Brasil \\ Greici Rössler Macuglia \\ Universidade Federal do Rio Grande do Sul \\ Porto Alegre, RS, Brasil \\ Cristiane Hammer \\ Martin Tesmmer \\ Universidade do Vale do Rio dos Sinos \\ São Leopoldo, RS, Brasil
}

\begin{abstract}
RESUMO
O uso de drogas na adolescência pode causar prejuízos ao desenvolvimento, podendo se estender ao longo da vida. A impulsividade é fator de risco para o consumo de álcool e outras drogas, podendo, assim, ser responsável tanto pelo início quanto pelo aumento da drogadição. O objetivo deste estudo foi verificar como e quando ocorre o início do uso de substâncias entre adolescentes do Rio Grande do Sul, investigando, também, os níveis de impulsividade e agressividade entre estes jovens, e se existem diferenças entre os sexos. Foram utilizados três instrumentos: (1) questionário sociodemográfico sobre uso de drogas; (2) escala de impulsividade de Barrat e (3) Inventário de Expressão de Raiva como Estado e Traço- STAXI. Conclui-se que o início do uso de substâncias tem ocorrido mais precocemente no Rio Grande do Sul do que em outros estados, e que as médias de impulsividade e agressividade destes adolescentes foram consideradas elevadas.
\end{abstract}

Palavras-chaves: Adolescência. Álcool e drogas. Impulsividade. Agressividade.

\begin{abstract}
Alcohol and Drugs Use, Levels of Impulsivity and Aggression in Adolescents in Rio Grande do Sul

Drug use in adolescence can cause damage to development, and may extend throughout life. Impulsivity is a risk factor for alcohol and other drugs, and may thus be responsible for both the beginning and the rise of drug addiction. The aim of this study was to determine how and when the onset of substance use among adolescents in Rio Grande do Sul, investigating also the levels of impulsivity and aggression among these young people, and whether there are differences between the sexes, Three instruments were used: (1) questionnaire on socio-demographic and drug use; (2) the Barratt Impulsiveness Scale, and (3) Anger Expression Inventory and State-Trait as STAXI. We conclude that the onset of substance use has occurred earlier in Rio Grande do Sul than in other states, and that the means of impulsivity and aggressiveness of these adolescents were considered elevated.
\end{abstract}

Keywords: Adolescents. Alcohol and drugs. Impulsivity. Aggressiveness.

\section{RESUMEN}

Consumo de Alcohol e Drogas, Niveles de Impulsividad y la Agresión de Adolescentes en Rio Grande do Sul

El consumo de drogas en la adolescencia puede causar daño al desarrollo, y se puede extender a lo largo de la vida. La impulsividad es un factor de riesgo de alcohol y otras drogas, y por lo tanto puede ser responsable de tanto el comienzo como el aumento de la drogadicción. El objetivo de este estudio fue determinar cómo y cuándo el inicio del consumo de drogas entre los adolescentes en Rio Grande do Sul, investigando también los niveles de impulsividad y la agresión entre los jóvenes, y si hay diferencias entre los sexos. Se utilizaron tres instrumentos: (1) socio-demográfica cuestionario sobre el consumo de drogas, (2) la Escala de Impulsividad de Barratt, y (3) de la ira Expresión y Estado-Rasgo como STAXI. Llegamos a la conclusión de que el inicio del consumo de sustancias se ha producido anteriormente en Rio Grande do Sul que en otros estados, y que los medios de la impulsividad y la agresividad de estos adolescentes se considera elevado.

Palabras clave: Adolescentes. Alcohol y drogas. Impulsividad. Agresividad. 
O uso de drogas inicia cada vez mais cedo, em adolescentes, fato que faz com que o uso precoce gere problemas quanto ao abuso e dependência de substâncias (Ferigolo et al., 2004; Strauch, Pinheiro, Silva e Horta, 2009; Galduróz, Noto, Fonseca e Carlini, 2004, Vieira et al., 2007).

A adolescência é um período marcado por mudanças, adaptações orgânicas e psíquicas (Vasters e Pillon, 2011), é um período de instabilidade, de busca de identidade, auto-afirmação e independência individual (Silva e Mattos, 2004), podendo ser uma fase decisiva em termos de desenvolvimento de competências, aquisição de habilidades pessoais, interpessoais e tomada de decisão (Vasters e Pillon, 2011).

É na adolescência que os indivíduos buscam novidades, constrõem suas identificações e, com isso, pode haver um distanciamento da família de origem e maior aproximação do grupo de iguais (Wagner, Falcke, Silveira e Mosmann, 2002). Afastar-se da família e aderir a um grupo de iguais (Knobel, 1992) pode representar um fator de risco, especialmente, quando o grupo é usuário de drogas. A família, pelo papel de inserir seus membros na cultura e ser instituidora das relações primárias, influencia a forma como o adolescente reage à ampla oferta de droga na sociedade. Relações familiares saudáveis desde o nascimento da criança podem servir como fator de proteção para toda a vida e, de forma muito particular, para o adolescente. Outros ambientes importantes para a prevenção do uso indevido de drogas são a escola, a comunidade e a mídia (Pechansky et al., 2004).

O álcool é uma das substâncias psicoativas mais consumidas pelos jovens e de consumo mais precoce, independente da classe econômica ou cultural (Galduróz, Noto, Fonseca e Carlini, 2004; Vieira et al., 2007; Vieira, 2008). Diferentes estudos confirmaram que o álcool pode ser facilmente obtido devido a frequente propaganda, o que reflete seu consumo precoce e disseminado (Pechansky et al., 2004; Soares, 2006).

A população jovem é vulnerável às consequências negativas e, muitas vezes, trágicas do uso de bebidas alcoólicas (Galduróz, Noto, Fonseca e Carlini, 2004). Nos Estados Unidos, o álcool está entre as quatro primeiras causas de morte entre indivíduos na faixa etária de 10 a 24 anos (acidentes de trânsito, ferimentos não intencionais, homicídio e suicídio) (NHTSA, 2001; McKinnon, O'rourke, Thompson, e Berumen, 2004; Pechansky et al., 2004). No Brasil, os problemas apontados variam desde acidentes de trânsito, comportamento sexual de risco (doenças sexualmente transmissíveis, gravidez indesejada) violência, ferimentos não intencionais e problemas acadêmicos (Reboussin, Song, Shrestha, Lohman e Wolfson, 2006; Laranjeira e Romano, 2004; Trindade e Correia, 1999).

O uso desta substância pode causar prejuízos ao desenvolvimento emocional e cognitivo dos jovens, que podem se estender ao longo da vida (Pechansky et al., 2004). Os seus efeitos repercutem na neuroquímica cerebral, em pior ajustamento social e no retardo do desenvolvimento de suas habilidades, já que um adolescente ainda está em processo de maturação em termos biológicos, sociais, pessoais e emocionais. Durante a adolescência o córtex pré-frontal ainda está em desenvolvimento, podendo o uso do álcool afetar uma série de habilidades que o adolescente necessita desenvolver e que são mediadas por este circuito (Pechansky et al., 2004; Strauch et al., 2009; Grant, 1998).

O consumo exagerado de álcool e de outras drogas na adolescência pode causar alterações neurofisiológicas profundas, causando graves danos à memória, ao aprendizado, à inteligência, à capacidade de abstração além de aumentar a propensão dos jovens ao alcoolismo (Pechansky et al., 2004; Buchalla, 2007; Paim, 2009).

Os adolescentes são particularmente susceptíveis às ofertas de droga (Dupont, 1987; Sanchez e Nappo, 2002; De Micheli e Formigoni, 2002), neste período a necessidade de autonomia leva o adolescente a rejeitar a proteção dos adultos e a enfrentar situações e condutas de risco (Vieira, 2008). Como consequência de toda a vulnerabilidade na qual se encontra o adolescente, devido as inúmeras transformações pelas quais passa, acaba sendo exposto a muitos riscos, dentre eles, o consumo de álcool e outras drogas. Dentre os fatores de risco para o uso abusivo de drogas, podemos citar: a disponibilidade das substâncias, as normas sociais; o uso de drogas ou atitudes positivas diante das drogas pela família, conflitos familiares graves (Almeida Filho, Ferreira, Gomes, Silva e Santos, 2007), entre outros.

A impulsividade, descrita como falta de planejamento, respostas muito rápidas aos estímulos, inabilidade de adiar gratificações e pobre controle inibitório, é também fator de risco para o desenvolvimento do consumo de álcool e outras drogas (Stoltenberg, Batiena e Birgenheir, 2008; Melo, 2001). A impulsividade e o uso de álcool e drogas são comorbidades, e podem ser responsáveis pelo início e pelo aumento da drogadição tanto quanto a drogadição pode elevar os níveis de impulsividade (Hogarth, 2011).

$\mathrm{O}$ abuso de drogas é considerado um ato impulsivo, mas ainda não está claro se essa característica impulsiva precede o uso da substância ou se é uma consequência 
do uso (Petry, Kirby e Kranzler, 2002; Scheffer e De Almeida, 2010), podendo ser a causa ou a decorrência do comportamento de adição (Wit, 2008; Scheffer e De Almeida, 2010).

Além da impulsividade, a agressividade também é um fator importante para o início e desenvolvimento do uso de substâncias, pois, existem evidências na literatura de que o comportamento violento está associado ao uso e/ou abuso do álcool (Meloni e Laranjeira, 2004).

No Rio Grande do Sul, não existem estimativas quanto ao número de adolescentes dependentes de álcool. Contudo, pesquisas realizadas pela Secretaria Nacional de Políticas sobre Drogas (SENAD) mostram que $7 \%$ dos jovens brasileiros, com idade entre $12 \mathrm{e}$ 17 anos consomem álcool, ou seja, 554.000 jovens são dependentes desta droga no país (SENAD, 2010). Quanto mais cedo se inicia o uso de álcool e tabaco, maior a vulnerabilidade de se desenvolver o abuso e a dependência das mesmas substancias e, concomitantemente, o uso de drogas ilícitas (Ferigolo et al., 2004; Heim, 2008).

Diante disto, o objetivo principal deste estudo foi verificar como e quando ocorre o início do uso de álcool e drogas entre os adolescentes de Porto Alegre e do interior do Rio Grande do Sul. Os objetivos específicos consistiram em investigar os níveis de impulsividade e agressividade destes jovens, e se existem diferenças entre adolescentes do sexo masculino e feminino no que concerne aos níveis de impulsividade e agressividade.

\section{MATERIAL E MÉTODOS}

A amostra estudada foi composta por 870 estudantes com média de idade de 13,19 anos (DP $=1,77$ anos), pertencentes a escolas públicas e privadas de Porto Alegre e do interior do estado do Rio Grande do Sul. Entre os jovens avaliados, 36,9\% eram do sexo masculino e $62,8 \%$ eram do sexo feminino. Os critérios de exclusão da amostra utilizados nesta pesquisa foram idade superior a 16 anos e inferior a 10 e uso de medicações psiquiátricas (Tabela 1).

TABELA 1

Dados sociodemográficos em porcentagem dos adolescentes pesquisados nas escolas públicas e privadas e o sexo

\begin{tabular}{cc}
\hline Escolas & Pesquisadas \\
Públicas & 16 \\
Privadas & 7 \\
\hline Sexo & $\%$ \\
Masculino & 36,9 \\
Feminino & 62,8 \\
\hline
\end{tabular}

Para a realização desta pesquisa, foram utilizados três instrumentos: o primeiro, um questionário socioeconômico, contendo respostas de escolha múltipla e, juntamente com esse questionário, um específico sobre o uso de álcool e de outras drogas, contemplando questões associadas ao início do uso deste tipo de substâncias (adaptado de Galduróz, Noto, Fonseca e Carlini, 2004). O segundo foi a Escala de Impulsividade de Barrat (BIS 11), que é uma escala autoaplicável composta de 30 itens com respostas do tipo Likert ${ }^{1}$ e que fornece um escore total de impulsividade e mais três subescores: (1) impulsividade motora, (2) de falta de planejamento e (3) atenção. Os escores variam de 30 a 120 e não há um ponto de corte estabelecido. Esta escala está validada para o uso em adolescentes (Diemen, 2006). O terceiro foi o Inventário de Expressão de Raiva como Estado e Traço (STAXI), utilizado com a finalidade de avaliar a agressividade através da mensuração de experiências e expressões de raiva (Spielberger, 1992). O instrumento foi traduzido e adaptado por Ângela M. B. Biaggio em 2003. A experiência de raiva no STAXI é avaliada como estado e traço. O Manual é composto por 44 itens e o formulário de aplicação possui três partes. A primeira, relativa à experiência de raiva como estado contém itens para o sujeito avaliar como está se sentindo no momento da aplicação; a segunda, relativa à experiência de raiva como traço, avalia como o sujeito se sente comumente, no seu dia a dia, sendo, portanto, mais ligada à personalidade; e a terceira que, por sua vez, se relaciona com o que o sujeito faz e o que sente quando está com raiva ou furioso, avaliando, portanto, a forma como este expressa sua raiva. As três partes do formulário apresentam coeficientes alfa maiores que 0,60 .

Para a realização dessa pesquisa, o grupo de pesquisadores entrou em contato com cada escola, explicando os objetivos da pesquisa e pedindo autorização para a realização da mesma com seus alunos. Uma vez autorizada, cada aluno recebia duas cópias do Termo de Consentimento Livre e Esclarecido para serem assinadas pelos pais e uma cópia, então, para ser devolvida para os pesquisadores a fim de documentar a autorização dos responsáveis para que o aluno participasse da pesquisa. Somente participaram da coleta aqueles alunos que trouxeram os termos assinados. A pesquisa passou pela aprovação do Comitê de Ética em Pesquisa do Instituto de Psicologia da Universidade Federal do Rio Grande do Sul. A partir da entrega dos termos assinados era dado início a aplicação dos testes nos alunos. As coletas foram feitas coletivamente, na sala de aula e em outros espaços físicos cedidos pela escola. As aplicações duravam em torno de uma hora e trinta minutos, podendo variar de acordo com a quantidade 
de alunos em cada aplicação. O período de duração da coleta de dados se estendeu de março de 2010 a dezembro de 2011, parando nos períodos de férias escolares.

\section{Análise dos dados}

Esta foi uma pesquisa quantitativa com caráter descritivo, de maneira que a análise dos dados consistiu em procedimentos descritivos para as variáveis de interesse (impulsividade e agressividade) e para as variáveis sociodemográficas e de características de uso de substâncias, envolvendo cálculo de médias e frequências das variáveis pesquisadas. Para a comparação entre os grupos foi utilizado o teste $t$ de Student. E, o grau de significância aceito foi $p<0,05$. Para a realização das análises estatísticas foi utilizado o software estatístico SPSS 18.0.

\section{RESULTADOS}

Os resultados obtidos indicaram que, dos jovens pesquisados, 63,6\% alegaram já ter feito uso de alguma bebida alcoólica, sendo que a média de idade do primeiro uso desta substância foi de 11,81 anos (Tabela 2).

Quanto ao tipo de bebida mais utilizada, 23,4\% dos jovens afirmaram ter bebido cerveja ou chope, seguidos por $14,2 \%$ que afirmaram ter utilizado destilados (cachaça, caipirinha, vodca, uísque e conhaque). Um dado importante a ser destacado, é que $26 \%$ dos jovens afirmaram terem experimentado bebida pela primeira vez em casa, sendo que quem mais tem oferecido bebida aos adolescentes são os familiares (Tabela 2).

TABELA 2

Porcentagem de adolescentes que experimentaram bebidas alcoólicas, qual bebida utilizada com mais frequência e quem ofereceu bebida pela primeira vez aos adolescentes

\begin{tabular}{lc}
\hline \multicolumn{1}{c}{ Uso de bebidas } & Frequência \\
\hline Utilizou bebida & \\
Sim & $63,6 \%$ \\
Não & $36,4 \%$ \\
Tipo de bebida & \\
Cerveja ou Chope & $23,4 \%$ \\
Destilados & $14,2 \%$ \\
Vinho & $12,4 \%$ \\
Champagne & $7,6 \%$ \\
Outros & $5,5 \%$ \\
Quem ofereceu & \\
Familiares & $26,4 \%$ \\
Amigos & $17,2 \%$ \\
Vontade própria & $6,2 \%$ \\
Não lembra & $10,6 \%$ \\
Outros & $3,1 \%$ \\
\hline
\end{tabular}

Com relação ao uso de outras drogas além do álcool, 14,4\% dos jovens pesquisados afirmaram já ter feito uso deste tipo de substâncias, e a média de idade do primeiro uso foi de 11,93 anos (Tabela 3). A droga mais utilizada pelos adolescentes foi o cigarro $(10,3 \%)$, seguido pela maconha $(3,1 \%)$. Os adolescentes relataram ter experimentado drogas pela primeira vez na casa de amigos/conhecidos $(5,1 \%)$, embora, na maior parte das vezes, as drogas foram oferecidas por desconhecidos $(8,2 \%)$ (Tabela 3$)$.

\section{TABELA 3}

Porcentagem quanto uso de outras drogas, droga mais utilizada, onde experimentou pela primeira vez e quem ofereceu a droga ao adolescente

\begin{tabular}{lc}
\hline \multicolumn{1}{c}{ Uso de drogas } & Frequência \\
\hline Utilizou drogas & \\
Sim & $14,4 \%$ \\
Não & $85,5 \%$ \\
Droga utilizada & \\
Cigarro & $10,3 \%$ \\
Maconha & $3,1 \%$ \\
Onde experimentou & \\
Em casa & $2,2 \%$ \\
Bar/festas & $2,2 \%$ \\
Casa de amigos/conhecidos & $5,1 \%$ \\
Não lembra & $2,9 \%$ \\
Outros & $2,4 \%$ \\
Quem ofereceu & \\
Vontade própria & $4,1 \%$ \\
Desconhecidos & $8,2 \%$ \\
Outros & $2,4 \%$ \\
\hline
\end{tabular}

Quanto aos níveis de agressividade, três domínios foram considerados: (i) Estado de Raiva, que diz respeito a como o adolescente se sentia no momento da avaliação, sendo algo mais instável; (ii) Traço de Raiva, que diz respeito aos aspectos mais da personalidade do adolescente, sendo mais estável ao longo do tempo; e (iii) Expressão de Raiva, que compreende a maneira que o adolescente expressa sua agressividade. Para estes três domínios, médias acima de 10 são considerados altos, indicando elevados níveis de agressividade. Com relação à Impulsividade, escores acima de 60 são consideradas altas, indicando altos níveis de impulsividade.

Os adolescentes que afirmaram já ter experimentado álcool alguma vez na vida obtiveram as seguintes médias: impulsividade, 66,78 (DP: 9,72) (Gráfico 1); agressividade traço, 22,11 (DP: 6,49); agressividade estado, 13,76 (DP: 5,14) e agressividade expressão, 


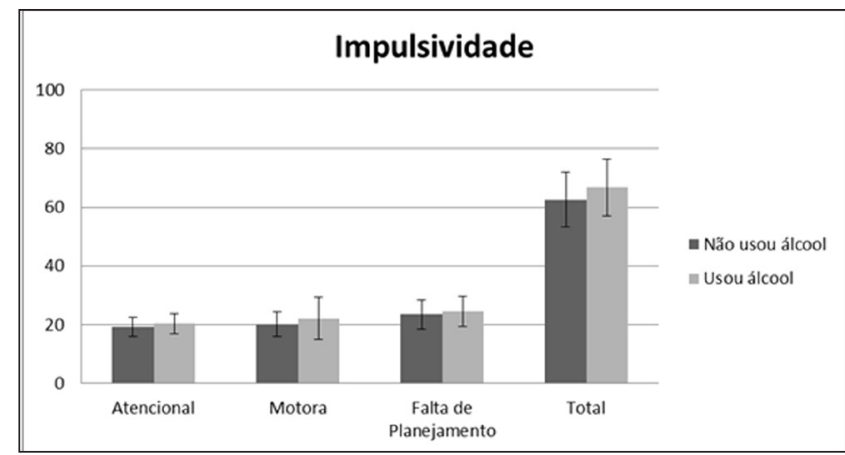

Gráfico 1. Níveis de Impulsividade entre adolescentes que utilizaram álcool e os que não utilizaram álcool

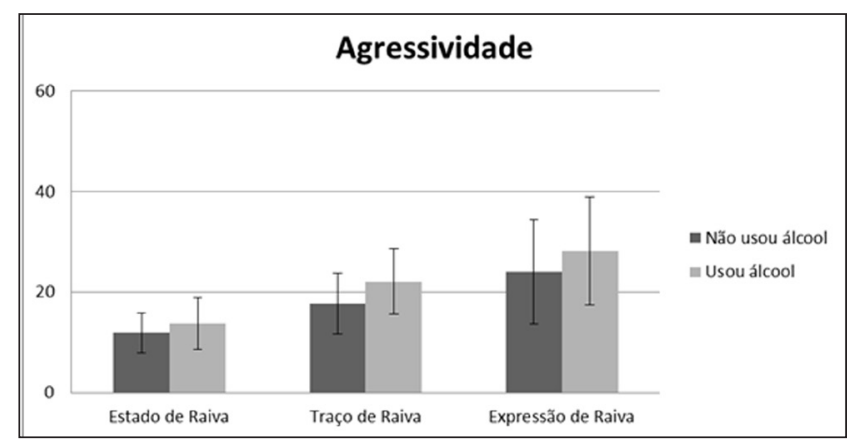

Gráfico 2. Níveis de Agressividade entre adolescentes que utilizaram álcool e os que não utilizaram álcool

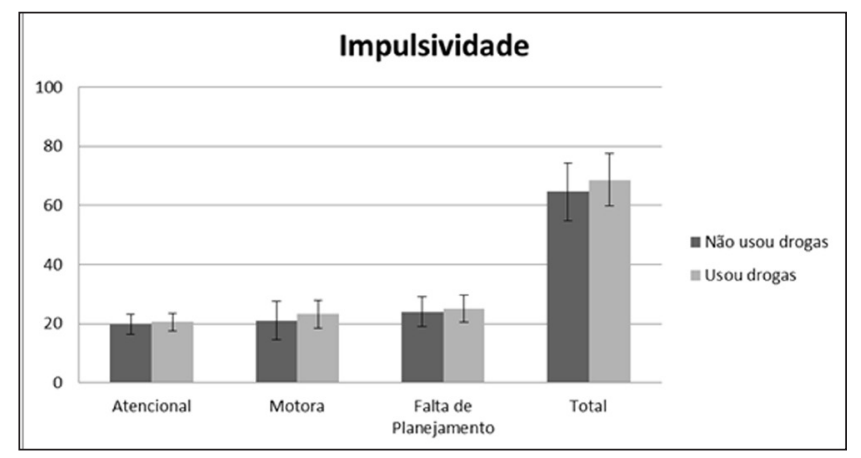

Gráfico 3. Níveis de Impulsividade entre adolescente que utilizaram outras drogas e os que não utilizaram

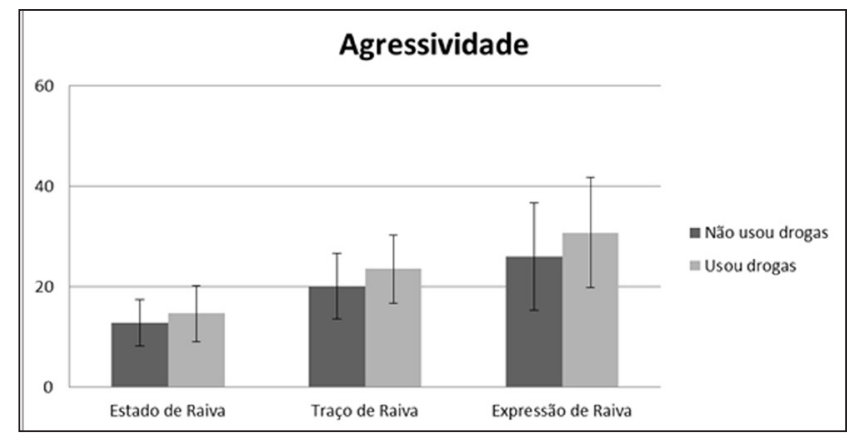

Gráfico 4. Níveis de Agressividade entre adolescentes que utilizaram outras drogas e os que não utilizaram outras drogas
28,18 (DP:10,82) (Gráfico 2). Já os adolescentes que afirmaram terem utilizado outras drogas, obtiveram como médias os seguintes valores: impulsividade, 66,70 (DP: 8,87) (Gráfico 3); agressividade traço, 23,52 (DP: 6,79); agressividade estado, 14,63 (DP: $5,61)$ e agressividade expressão, 30,80 (DP: 10,90) (Gráfico 4).

A partir da análise dos resultados pode-se perceber que os adolescentes pesquisados obtiveram médias consideradas altas nos três domínios da agressividade e, também, na impulsividade, evidenciando altos níveis de impulsividade e agressividade, que podem de certa forma, explicar o início precoce do uso deste tipo de substâncias.

Com relação às diferenças entre meninos e meninas no que diz respeito aos níveis de impulsividade e agressividade, foram verificadas diferenças estatisticamente significativas entre os dois sexos quanto à agressividade no Estado e noTraço de Agressividade, $p<0,05$ e $p<0.01$, respectivamente (Tabela 4).

\section{TABELA 4}

Diferenças nos níveis de impulsividade e agressividade entre meninos e meninas

\begin{tabular}{|c|c|c|c|c|c|c|}
\hline \multirow{2}{*}{ Sexo } & \multicolumn{2}{|c|}{ Meninos } & \multicolumn{2}{|c|}{ Meninas } & \multirow{2}{*}{$p^{*}$} & \multirow{2}{*}{$t$} \\
\hline & $M$ & $D P$ & $M$ & $D P$ & & \\
\hline Estado de raiva & 13,96 & 0,31 & 12,86 & 0,51 & 0,05 & $-1,78$ \\
\hline Traço de raiva & 19,81 & 1,04 & 21,00 & 0,98 & 0,01 & 2,53 \\
\hline Expressão de raiva & 26,67 & 10,58 & 26,69 & 11,03 & 0,98 & 0,20 \\
\hline Total impulsividade & 65,15 & 9,27 & 65,28 & 10,13 & 0,85 & 0,18 \\
\hline
\end{tabular}

\section{DISCUSSÃO}

A partir dos dados coletados verificou-se que o início do uso de álcool em adolescentes do Rio Grande do Sul que é de 11,81 anos, ocorre mais precocemente, do que no restante do Brasil, onde de acordo com o VI levantamento Nacional sobre o consumo de drogas entre estudantes do ensino fundamental e médio da rede pública e privada nas capitais brasileiras, realizado em 2010 pelo Centro Brasileiro de Informações sobre Drogas Psicotrópicas (CEBRID) ocorre em média aos 13 anos de idade. Este resultado é preocupante, pois esses adolescentes podem ter um maior risco de desenvolver dependência na fase adulta (Meloni e Laranjeira, 2004; Vieira, Ribeiro, Romano e Laranjeira, 2007).

Quando comparadas as médias de idade do início do uso de álcool e drogas entre meninos e meninas verificou-se que não houve diferença significativa entre os grupos, dados estes também evidenciados por outras pesquisas (Tavares, Béria e Lima, 2001; 
Strauch et al., 2009). Isso se torna preocupante porque as mulheres apresentam alguns fatores que as tornam mais vulneráveis aos efeitos do álcool, como menos desidrogenase (metabolizador do álcool), menor peso, mais gordura corporal que retém o álcool, por mais tempo, e as variações do ciclo hormonal (Martins, 2006). Laranjeira e Pinsky (2000), alertam que essa mudança no comportamento do beber das mulheres pode ser devido à mudança no status e na sua participação na sociedade, pois em décadas passadas não era socialmente aceitável que a mulher exagerasse ou perdesse o controle sobre os seus atos.

Outro resultado encontrado que merece destaque é que os familiares são os que mais oferecem álcool aos adolescentes, seguido por amigos, considerando o primeiro uso, dado este que aparece em outras pesquisas (Vieira et al., 2007; Martins, 2006; Dallo, 2011). Estudos mostram que o uso nocivo de álcool pelos pais e a falta de controle e vigilância sobre consumo dos filhos estão associados com o aumento do risco de abuso e dependência de álcool mais tarde na vida e que pode ter uma questão cultural presente (Grunbaum, 2003; Kustsche, 2004; Vieira, 2007).

Conforme Edwards, Marshal e Cook (2005), a cultura pode trazer influências ao padrão, contexto e quantidade de consumo de álcool, além da cultura familiar também ser outro fator determinante no ato de beber. É provável que os filhos possam herdar dos pais padrões excessivos de beber, o comportamento de beber é assimilado e são atribuídos valores e crenças associados ao ato de beber. Embora, o uso de álcool seja considerado pela família uma atitude negativa para o desenvolvimento dos filhos, gerando risco de se tornarem usuários, pesquisas apontaram que atitudes permissivas dos pais são consideradas maior fator de risco nesta situação (Schenker e Minayo, 2005; Swadi, 2000).

Outro fator de risco que está relacionado ao consumo precoce de álcool pelos adolescentes é a publicidade de bebidas alcoólicas (Faria, 2011; Henriksen, 2007; Stacy, 2004). Diversos estudos têm apontado essa influência da propaganda no consumo (Faria, 2011; Fleming, 2004; Babor, 2003), bem como o fácil acesso dos adolescentes a essa droga (Silva, 2006), corroborando com os resultados desta pesquisa onde a maioria dos adolescentes respondeu que não encontram dificuldades em obter o álcool.

Quanto ao consumo de outras drogas $14,4 \%$ dos adolescentes já utilizaram, sendo que a média de idade do primeiro uso foi de 11,93 anos. Entre as drogas mais utilizadas estão o cigarro $10,3 \%$ e a maconha $3,1 \%$. Embora os adolescentes relatem que fizeram uso de drogas na casa de amigos, estas thes são oferecidas por desconhecidos. Pesquisas evidenciam que $80 \%$ do adultos fumantes iniciaram este hábito antes dos 18 anos de idade (WHO, 2009; Rodrigues, 2009), o que demonstra uma necessidade urgente de se trabalhar a prevenção. Ressaltamos que não houve diferenças significativas entre gêneros, resultado que confirmam outros estudos (Granville-Garcia et al., 2011; Fraga, 2006).

Os jovens apresentam um risco mais alto para desenvolver dependência química e outros comportamentos de risco em função da impulsividade, pois as estruturas cerebrais responsáveis pelo controle dos comportamentos e dos impulsos ainda estão em fase de maturação (Stoltenberg et al., 2008), o que faz com que os adolescentes tendam a valorizar muito mais o presente, sejam mais imediatistas e não pensem tanto nas consequências de seus atos.

Os níveis de Impulsividade e Agressividade encontrados entre os adolescentes pesquisados que afirmaram ter utilizado álcool e/ou drogas foram considerados altos, fato corroborado por pesquisas que afirmam que aumentos na quantidade de ingestão de álcool são concomitantemente associados a aumentos no comportamento agressivo e vice-versa (White, Fite, Pardini, Mun e Loeber, 2012).

O comportamento agressivo tem sido apontado como um comportamento de risco (Chiapetti, 2001; Werner e Nixon, 2005; Barnow, Lucht e Freyberger, 2005; Guimarães, 2006) e na visão de Kaplan, Sadock e Grebb (1997), o comportamento agressivo estaria relacionado a conflitos despertados pelo ambiente interpessoal, que, associados ou não a um comprometimento orgânico ou neurológico, agem de forma a fortalecer os impulsos agressivos. Tais impulsos, quando não regulados devido a uma baixa capacidade de autocontrole (fragilidade intrapsíquica), podem dar origem a comportamentos de risco psicossocial, sobretudo com manifestações de violência. $\mathrm{O}$ álcool e outras substâncias químicas funcionam como facilitadores de situações de violência (Heim, 2008).

Tivemos algumas limitações durante a pesquisa, a principal foi a dificuldade de acesso às escolas, principalmente, particulares. Foi negado acesso à pesquisa e alegado que na escola não havia problema com drogas, ou que assuntos tidos como "tabus", por exemplo, drogas e sexo, não eram abordados para não despertarema curiosidade dos alunos.

\section{CONCLUSÕES}

Este estudo evidenciou que o início do uso de álcool e outras drogas estão acontecendo precocemente no Rio Grande do sul, estando abaixo da 
média nacional. Nos adolescentes que fizeram uso destas substâncias os níveis de agressividade e impulsividade foram superiores aos adolescentes que não utilizaram. Os familiares, principalmente pais, são os responsáveis pelo primeiro uso do álcool, sendo eles que proporcionam este primeiro contato. Fazem-se necessários programas de prevenção para trabalhar tanto com os pais, professores e com os adolescentes.

\section{REFERÊNCIAS}

Almeida Filho, A. J., Ferreira, M. A., Gomes, M. L. B, Silva, R. C., \& Santos, T. C. F. (2007). O adolescente e as drogas: consequências para a saúde. Escola Anna Nery Revista de Enfermagem, 11(4), 605-610.

Babor, T., Caetano, R., Casswell, S., Edwads, G., \& Giesbrecht, N. (2003). Alcohol, no ordinary commodity: research and public policy. New York: Oxford University Press; Regulating alcohol promotion, 173-188.

Barnow, S., Lucht, M., \& Freyberger, H. (2005). Correlates of aggressive and delinquent aonduct problems in adolescence. Aggressive/Behavior, 31(1), 24-39.

Cohen, P., Cohen, J., Kasen, S., Velez, C. N., Hartmark, C., \& Johnson, J. (1993). An epidemiologic study of disorders in late childhood and adolescence, I: age and gender-specific prevalence. Journal Child Psychology Psychiatry, 34, 851-867.

Dallo, L., \& Martins, R. A. (2011). Uso de álcool entre adolescentes escolares: um estudo-piloto. Paidéia (Ribeirão Preto), 21(50), 329-334.

Chiapetti, N. (2001). Comportamento de risco em pré-adolescentes institucionalizados. Tese de Doutorado não-publicada. Programa de Pós-Graduação em Psicologia Clínica do Instituto de Psicologia da Universidade de São Paulo, São Paulo.

Diemen, L. von (2006). Associação entre impulsividade, idade do primeiro consumo de álcool e abuso de substâncias psicoativas em adolescentes de uma região do Sul do Brasil. Tese de Doutorado. Programa de Pós-Graduação da Faculdade de Medicina, Universidade Federal do Rio Grande do Sul. Porto Alegre. Acesso em 20 mar. 2009, de http://www.lume.ufrgs. $\mathrm{br} /$ bitstream/handle/10183/10073/000594494.pdf? sequence $=1$

Dupont, R. L. (1987). Prevention of adolescent chemical dependency. Pediatric Clinics of North America, 34(2), 495- 505.

Edwards, G., Marshall, E. J., \& Cook, C. C. H. (2005). O tratamento do alcoolismo: um guia para profissionais da saúde ( $4^{\mathrm{a}}$ ed.). Porto Alegre: Artmed.

Faria, R., Vendrame, A., Silva, R., Pincy, I. (2011). A propaganda de álcool e associação ao consumo de cervejas por adolescentes. Revista de Saúde Pública, 45(3), 441-447.

Ferigolo, M., Barbosa, F. S., Arbo, E., Malysz, A. S., Stein, A. T. \& Barros, H. M. T. (2004). Drug prevalence at Febem. Revista Brasileira de Psiquiatria, 26(1), 9-15.

Fleming, K., Thorson, E., \& Atkin, C. K. (2004). Alcohol advertising exposure and perceptions: links with alcohol expectancies and intentions to drink or drinking in underaged youth and young adults. J Health Commun, 9(1), 3-29.

Fraga, S., Ramos, E., \& Barros, H. (2006). Uso de tabaco por estudantes adolescentes portugueses e fatores associados. Revista de Saúde Püblica, 40(4), 620-626.

Galduróz, J. C. F., Noto, A. R., Fonseca, A. M., \& Carlini, E. A. (2004). V Levantamento Nacional sobre o Consumo de Drogas
Psicotrópicas entre Estudantes do Ensino Fundamental e Médio da Rede Pública de Ensino nas 27 Capitais Brasileiras. In CEBRID, São Paulo, Brasil. Acesso em 16 de maio de 2012, de http://200.144.91.102/cebridweb/download.aspx?cd=106

Galduroz, J. C., Noto, A. R., Nappo, S. A., Carlini, E.A. (2004). Trends in drug use among students in Brazil: analysis of four surveys in 1987, 1989, 1993 and 1997. Brazilian Journal of Medical and Biological Research, 37(4), 523-531.

Grant, B. R. (1998). The impact of a family history of alcoholism on the relationship between age at onset of alcohol use and DSMIV alcohol dependence: Results of the National Longitudinal Alcohol Epidemiologic Survey. Alcohol Health \& Reserach World, 22(2), 144-147.

Grunbaum, J. A., Kann, L., Kinchen, S., Ross, J., Hawkins, J., Lowry, R., et al. (2004). Youth risk behavior surveillance-United States, 2003. MMWR Surveill Summ, 53(2), 1-96.

Guimarães, N. M., Pasian, S. R. (2006). Agressividade na adolescência: Experiência e Expressão de Raiva. Psicologia em Estudo (Maringá), 11(1), 89-97.

Hogarth, L. (2011). The role of impulsivity in the etiology of drug dependence reward sensitivity versus automaticity. Psychopharmacology, 215(3), 567-580.

Heim, J., Andrade, A. G. (2012). Efeitos do uso do álcool e das drogas ilícitas no comportamento de adolescentes de risco: uma revisão das publicações científicas entre 1997 e 2007. Revista de Psiquiatria Clínica, 35(1), 61-64.

Henriksen, L., Feighery, E. C., Schleicher, N. C., Fortmann, S. P. (2008). Receptivity to alcohol marketing predicts initiation of alcohol use. J Adolesc Health, 42(1), 28-35.

Kaplan, H. I., Sadock, B. J., \& Grebb, J. A. (1997). Contribuições das ciências sociais para o comportamento humano. In H. I. Kaplan, B. J. Sadock, \& J. A. Grebb (Eds.). Compêndio de psiquiatria: ciências do comportamento e psiquiatria clínica (7 $7^{\mathrm{a}}$ ed) (pp. 170-178). Porto Alegre: Artes Médicas.

Knobel, M. (1992). A síndrome da adolescência normal. In A. Aberastury, \& M. Knobel (Eds.). Adolescência normal: um enfoque psicanalítico. Porto Alegre: Artes Médicas.

Kuntsche, E., Rehm. J., Gmel. G. (2004). Characteristics of binge drinkers in Europe. Social Science \& Medicine, 59(1), 113-127.

Laranjeira, R., \& Pinsky, I. (2000). Alcoolismo (6a ed.). São Paulo: Contexto

Laranjeira R., \& Romano M. (2004). Consenso brasileiro sobre políticas públicas do álcool. Revista Brasileira de Psiquiatria, 26(1), 68-77.

Martins, R. A. (2006). Uso de álcool, intervenção breve e julgamento sócio-moral em adolescentes que bebem excessivamente. Tese de Livre Docência não publicada, Universidade Estadual Paulista, São José do Rio Preto, SP.

McKinnon, S. A., O'rourke, K. M., Thompson, S. E., \& Berumen, J. H. (2004). Alcohol use and abuse by adolescents: the impact of living in a border community. $J$ Adolesc Health, 34(1), 88-93.

Melo, J. M. (2001). O alcoolismo no universo TEEN: Mídia e saúde. São Paulo: Universidade Metodista, 747-766.

Meloni, J. N., \& Laranjeira, R. (2004). Custo social e de saúde do uso do álcool. Revista Brasileira de Psiquiatria, 26(1), 7-10.

De Micheli D., \& Formigoni M. L. (2002). Are reasons for the first use of drugs and family circumstances predictors of future use patterns? Addictive Behaviors, 27(1), 87-100.

National highway traffic safety administration (NHTSA). Traffic Safety Facts 2000: Young drivers DOT HS-809-336. Washington, DC: NHTSA; 2001.

Paim, M. (2009). Alcoolismo: um alerta aos jovens. Missão jovem: Renova-se a esperança. Atualidades, ano XXVII(241). 
Pechansky, F., Szobot, C. M., Scivoletto, S., (2004). Uso de álcool entre adolescentes: conceitos, características epidemiológicas e fatores etiopatogênicos. Revista Brasileira de Psiquiatria, 26(1), 14-17.

Petry, N. M., Kirby, K. N., \& Kranzler, H. R. (2002). Effects of gender and family history of alcohol dependence on a behavioral task of impulsivity in healthy subjects. Journal of Studies on Alcohol, 63(1), 83-90.

Reboussin, B. A., Song, E. Y., Shrestha, A., Lohman, K. K., Wolfson, M. (2006). A latent class analysis of underage problem drinking: evidence from a community sample of 16-20 year olds. Drug Alcohol Dependence, 83(3), 199-209.

Rodrigues, M.C., Viegas, C. A. A., Gomes, E. L., Morais, J. P. M. G., \& Zakir, J. C. O. (2009). Prevalência do tabagismo e associação com o uso de outras drogas entre escolares do Distrito Federal. J Bras Pneumol, 35(10), 986-991.

Sanchez Z. M., \& Nappo S. A. (2002). A seqüência de drogas consumidas por usuários de crack e fatores interferentes. Revista de Saúde Pública, 36(4), 420-430.

Scheffer, M., \& Almeida, R.M.M. de. (2010). Consumo de álcool e diferenças entre homens e mulheres: comportamento impulsivo, aspectos cognitivos e neuroquímicos. Revista de Neuropsicologia Latinoamericana, 2(3), 1-11.

Schenker, M., \& Minayo, M.C.S. (2005). Fatores de risco e de proteção para o uso de drogas na adolescência. Ciência \& Saúde Coletiva, 10(3), 707-717.

Silva, V. A., \& Mattos, H. F. (2004). Os jovens são mais vulneráveis às drogas? In I. Pinsky, \& M. A. Bessa (Eds.). Adolescência e drogas. São Paulo: Contexto.

Spielberger, C. D., \& Biaggio, A. (1992/2003). Manual do STAXI. São Paulo: Vetor.

Silva, E. F, Pavani, R. A. B., Moraes, M. S., \& Neto, F. C. (2006). Prevalência do uso de drogas entre escolares do ensino médio do município de São Jose do Rio Preto, São Paulo, Brasil. Cad Saude Publica, 22(6), 1151-1158.

Soares, R. (2006). Inimigo íntimo: o álcool e o cérebro dos jovens. Revista Veja (São Paulo), abril, 96-104.

Stacy, A. W., Zogg, J. B., Unger, J. B., \& Dent, C. W. (2004). Exposure to televised alcohol ads and subsequent adolescent alcohol use. Am J Health Behav, 28(6), 498-509.

Stoltenberg, S. F., Batiena, B. D., \& Birgenheir, D. G. (2008). Does Gender Moderate Associations Among Impulsivity and HealthRisk Behaviors? Addict Behavior, 33(2), 252-265.

Strauch, E. S. Pinheiro, R. T., Silva, R. A., \& Horta, B. L. (2009). Uso de álcool por adolescentes: estudo de base populacional. Revista de Saúde Pública, 43(4), 647-655.

Swadi, H. (2000). Individual risk factors for adolescent substance use. Drug and Alcohol Dependence, 55(3), 209-224.

Tavares, B. F., Béria, J. U., \& Lima, M. S. (2001). Prevalência do uso de drogas e desempenho escolar entre adolescentes. Rev Saude Publica, 35(2), 150-158.

Trindade, I., \& Correia, R. (1999). Adolescentes e álcool: estudo do comportamento de consumo de álcool na adolescência. Análise Psicológica, 17(3).
Vasters, G. P., \& Pillon, S. C. (2011). Drugs use by adolescents and their perceptions about specialized treatment adherence and dropout. Revista Latino-Americana de Enfermagem, 19(2), 317-324.

Vieira, P. C. (2008). Uso de álcool, tabaco e outras drogas por adolescentes escolares em município do sul do Brasil. Caderno de Saúde Pública, Rio de Janeiro, 24(11), 2487-2498.

Vieira, D. L., Ribeiro, M., Romano, M., \& Laranjeira, R. R. (2007). Alcohol and adolescents: study to implement municipal policies. Revista Saúde Pública, 41(3), 396-403.

Wagner, A., Falcke, D., Silveira, L. M. B. O., \& Mosmann, C. P. (2002). A comunicação em famílias com adolescentes. Psicologia em Estudo, 7(1), 75-80.

Wit, H. (2008). Impulsivity as a determinant and consequence of drug use: A review of underlying processes. Addiction Biology, 14(1), 22-31.

Werner, N. E., \& Nixon, C. L. (2005). Normative beliefs and relational aggression: An investigation of the cognitive bases of adolescent aggressive behavior. Journal of Youth and Adolescence, 34(3), 229-243.

World Health Organization [homepage on the Internet]. Geneva: World Health Organization [cited 2009 Feb 10]. International Consultation on Tobacco and Youth - What in the World Works? Available from: http://www.who.int/tobacco/dy_speeches7/en/

Nota:

1 Essa mensuração é muito utilizada nas ciências sociais, especialmente em levantamento de atitudes, opiniões e avaliações. Nela pede-se ao respondente que avalie um fenômeno numa escala de alternativas, as afirmações podem ser auto-referentes (Günther, 2003).

\section{Autores:}

Rosa Maria Martins de Almeida - Professora Adjunta do Instituto de Psicologia do Desenvolvimento e da Personalidade da Universidade Federal do Rio Grande do Sul (UFRGS), Porto Alegre, RS, Brazil. Laboratório de Psicologia Experimental, Neurociências e Comportamento. Pesquisadora de Produtividade do CNPq. Pesquisa financiada pelo CNPq.EPe

Laís Broch Trentini - Aluna do Curso de Graduação de Psicologia da Universidade Federal do Rio Grande do Sul. Bolsista de Iniciação Científica do CNPq.

Lidiane Andreza Klein - Aluna do Curso de Graduação em Psicologia Habilitação e Formação de Psicólogos pela Universidade do Vale do Rio dos Sinos. Bolsista de Apoio Técnico do CNPq.

Greici Rössler Macuglia - Aluna de Doutorado do Curso de Pós-Graduação em Psicologia da Universidade Federal do Rio Grande do Sul.

Cristiane Hammer - Aluna do Curso de Graduação em Psicologia Habilitação e Formação de Psicólogos pela Universidade do Vale do Rio dos Sinos.

Martin Tesmmer - Aluno do Curso de Graduação em Psicologia Habilitação e Formação de Psicólogos pela Universidade do Vale do Rio dos Sinos.

\section{Endereço para correspondência:}

Rosa Maria Martins de Almeida

Instituto de Psicologia - Universidade Federal do Rio Grande do Sul

Rua Ramiro Barcelos, 2600 - Santa Cecília

90035-003 Porto Alegre, RS, Brasil

Tel.: (51)3308-5066 - Fax: (51)3308-5470

E-mail: rosa_almeida@yahoo.com or rosa.almeida@ufrgs.br

Recebido em: 01.12.2012.

Aceito em: 02.04.2013. 